\title{
Production and propagation of heavy hadrons in air-shower simulators
}

\author{
C. A. García Canal ${ }^{1}$, J. I. Illana², M. Masip ${ }^{2}$, S. J. Sciutto ${ }^{1}$ \\ ${ }^{1}$ IFLP/CONICET and Departamento de Física \\ Universidad Nacional de La Plata, C.C.67, 1900, La Plata, Argentina \\ ${ }^{2}$ CAFPE and Departamento de Física Teórica y del Cosmos \\ Universidad de Granada, E-18071, Granada, Spain \\ cgarciacanal@fisica.unlp.edu.ar, jillana@ugr.es, masip@ugr.es, \\ sciutto@fisica.unlp.edu.ar
}

\begin{abstract}
Very energetic charm and bottom hadrons may be produced in the upper atmosphere when a primary cosmic ray or the leading hadron in an extensive air shower collide with a nucleon. At $E \approx 10^{8} \mathrm{GeV}$ their decay length becomes of order $10 \mathrm{~km}$, implying that they tend to interact in the air instead of decaying. Since the inelasticity in these collisions is much smaller than the one in proton and pion collisions, there could be rare events where a heavy-hadron component transports a significant amount of energy deep into the atmosphere. We have developed a module for the detailed simulation of these processes and have included it in a new version of the air shower simulator AIRES. We study the frequency, the energy distribution and the depth of charm and bottom production and decay in the atmosphere. As an illustration, we consider the production and decay of tau leptons (from $D_{s}$ decays) and the lepton flux at $\mathrm{PeV}$ energies from a $30 \mathrm{EeV}$ proton primary. The proper inclusion of charm and bottom hadrons in AIRES opens the possibility to search for air-shower observables that are sensitive to heavy quark effects.
\end{abstract}




\section{Introduction}

Air-shower simulations are an essential tool in cosmic-ray physics [1, 2]. Primary particles reach the Earth with energies of up to $10^{11} \mathrm{GeV}$, in particular, AUGER is exposed to around 15,000 events of energy above $10^{10} \mathrm{GeV}$ (10\% of them hybrid) per year [3]. Such energies are well above the ones explored at colliders, and the simulation of these events requires then an extrapolation of the known physics that could be affected by several factors. On one hand, there could be new particles or interactions not accessible at lower energies. In this sense, cosmic rays may offer opportunities in the search for strong $\mathrm{TeV}$ gravity [7], new neutrino interactions [8], or long-lived massive particles [9]. On the other hand, cosmic-ray energies may imply a regime where the properties of standard particles can be substantially different. Consider, in particular, the hadrons containing a charm or a bottom quark, whose properties are well known from collider experiments. The lightest mode with a given quark content will always decay through a weak interaction, implying a relatively long lifetime $(c \tau=0.1-$ $0.5 \mathrm{~mm}$ ). Although at the Tevatron or the LHC very energetic heavy hadrons may define events with observable displaced vertices, such hadrons will never reach the calorimeters there. In contrast, when produced with energies above $10^{8} \mathrm{GeV}$ inside an extensive air shower (EAS) their decay length becomes larger than $10 \mathrm{~km}$, and they tend to collide in the atmosphere instead of decaying. The collisions with matter of these long-lived heavy hadrons would introduce physics unseen at colliders. This physics will certainly occur in extensive air showers, and its inclusion in the simulation may be necessary to explain rare effects or just as a standard background in the search for genuine exotics.

The heavy quark inside an ultrarelativistic $D$ or $B$ meson carries most of the hadron energy. If the meson collides with an air nucleus and breaks into several pieces, it is obvious that the piece carrying the heavy quark will take most of the energy after the collision. Therefore, at $E>10^{8} \mathrm{GeV}$ these particles become long lived and much more penetrating than a proton or a pion: a simulation seems necessary to establish whether heavy mesons are effective in taking a significant fraction of this energy deep into the atmosphere.

In addition, at energies above $100 \mathrm{GeV}$ pions and kaons become less effective producing atmospheric muons and neutrinos (they tend to collide with air nuclei instead of decaying), and the spectral index in the lepton flux that they yield is reduced in one unit [10]. The prompt decay of charmed hadrons has been extensively studied [11] as the dominant source of leptons at PeV energies (see also [12]). The non-prompt charm contribution (from charm decaying after one or several collisions in the air), however, may be not negligible, specially at higher energies, and its inclusion in the simulation requires an estimate of propagation effects.

In this article we report on the inclusion of heavy-quark production and propagation in 
the air-shower simulator AIRES [1]. AIRES provides full space-time shower development in a realistic environment, taking into account the atmospheric density profile, the Earth's curvature, and the geomagnetic field. The new version of the simulator used in this work recognizes and propagates photons, electrons, positrons, muons, neutrinos, pions, kaons, eta mesons, lambda baryons, nucleons, antinucleons, nuclei up to $Z=36$, as well as $D$ and $B$ mesons, $\Lambda_{c}$ baryons, and tau leptons (which may appear in $D_{s}$ decays). AIRES is able to process complex decay schemes with a large number of branches, as it is the case for heavy hadrons. Nucleus-nucleus, hadron-nucleus, and photon-nucleus inelastic collisions with significant cross-sections are taken into account via calls to external hadronic packages.

For the present work we have developed a hadronic interaction preprocessor (HQIP, for Heavy Quark Interaction Preprocessor) that simulates collisions including heavy hadrons. The algorithms used in HQIP are based on a perturbative QCD framework that is described in some detail in section 2. When processing high energy hadronic collisions, HQIP complements the usual hadronic packages (SIBYLL [4] or QGSJET [5]). If HQIP is invoked the charm production option in the external hadronic package is set to disabled. The production of heavy quarks in nucleus-nucleus and photon-nucleus collisions has not been implemented yet.

The impact of charm production in air showers has been previously discussed in [6]. That work, however, does not include bottom quark production nor the propagation of heavy hadrons in the atmosphere (i.e., their possible collisions with air nuclei).

This article is organized as follows. In section 2 we discuss the production cross sections and the inelasticities in heavy meson collisions with air nuclei that we have used. In section 3 we illustrate the performance of AIRES with a study of the energy and depth distributions of heavy hadron production and decay for a vertical proton primary of fixed $30 \mathrm{EeV}=$ $3 \times 10^{10} \mathrm{GeV}$ energy. Finally, we discuss the spectrum of muons and the production of very energetic tau leptons from heavy quark decays for the same proton primary.

\section{Charm and bottom production and propagation}

Heavy-quark production by cosmic rays has been considered by a number of groups (see [13] for a review). At ultrahigh energies the usual calculation of the partonic process using perturbative QCD is very dependent on the small- $x$ gluon distribution, giving results that can vary by more than an order of magnitude [11]. Instead, we will base our cross sections on the color dipole picture [14] described in detail in [15, 16], which incorporates in a simple way saturation and nuclear effects and yields much smaller uncertainties. Within this picture a gluon carrying a fraction $x_{1}$ of the projectile energy fluctuates into a $Q \bar{Q}$ dipole that 

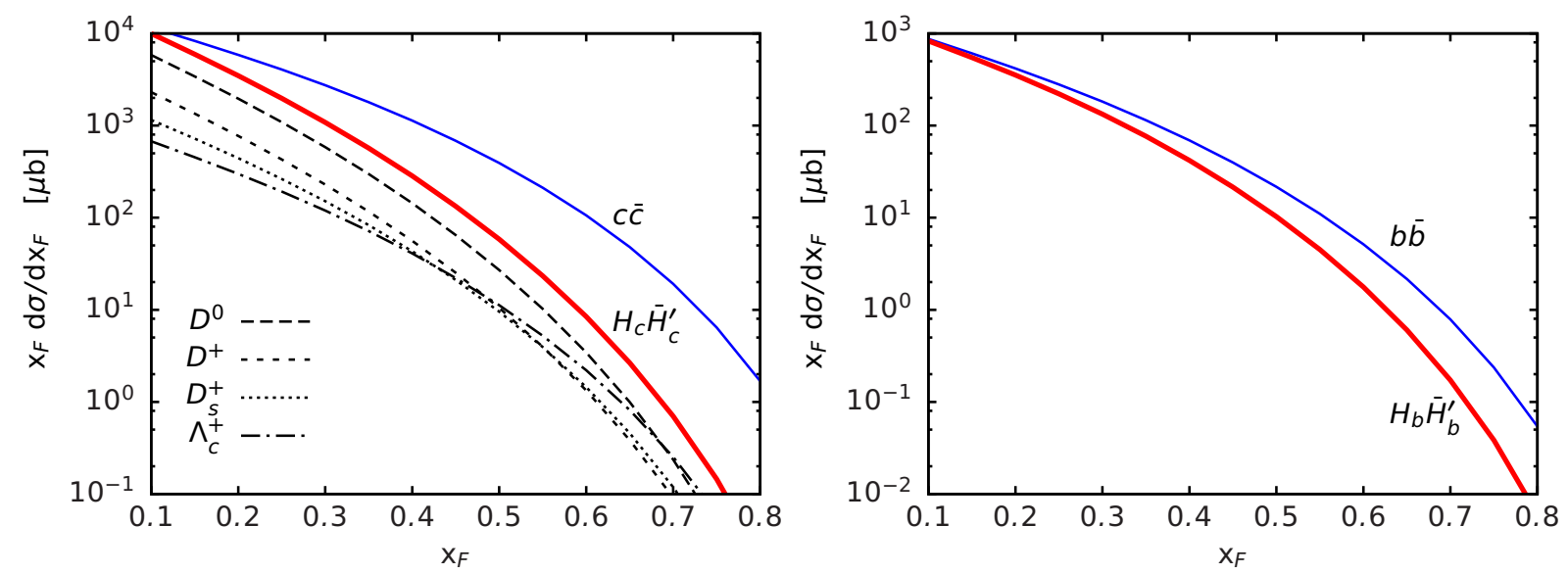

Figure 1: Differential cross section for $c \bar{c}$ (left) and $b \bar{b}$ (right) production in $p$-air collisions for an incident energy of $10^{10} \mathrm{GeV}$. The upper line gives the fraction $x_{F}$ of energy taken by the $Q \bar{Q}$ pair, whereas the lower (thick) line gives the energy taken by the two hadrons after fragmentation. In dashes we plot the cross section for the production of each charmed species.

interacts coherently with the gluon field in the target (the $x_{2} \ll 1$ gluons form a Color Glass Condensate) and evolves into hadrons. GM [15] and ERS [16] provide the inclusive differential cross section $\mathrm{d} \sigma^{Q \bar{Q}} / \mathrm{d} x_{F}$ in proton-air collisions, where the Feynman variable $x_{F}=x_{1}-x_{2} \approx x_{1}$ gives the fraction of incident energy taken by the heavy-quark pair. Their results agree within the $50 \%$ uncertainty that is expected from the choice of scales, PDFs, charm (or bottom) quark mass, and nuclear effects (see Fig. 3 in [16]). The new version of AIRES uses the simple parametrization in [15]. We plot in Fig. 1 (upper lines) the differential cross section for a $10^{10} \mathrm{GeV}$ incident proton. The pair energy $\left(x_{F} E\right)$ will be distributed between the two heavy quarks. In particular, we assume that the fraction $y$ of energy taken by each quark follows a flat distribution between $y_{\min , \max }=0.5\left(1 \mp \sqrt{1-4 \epsilon^{2}}\right)$, with $\epsilon=m_{Q} / m_{Q \bar{Q}} \approx 0.3$.

The heavy quarks will then fragment into hadrons. For the charm quark we have used the Kniehl and Kramer parametrization [17], including the fragmentation into $D^{0}, D^{+}$and $D_{s}^{+}$ mesons and $\Lambda_{c}^{+}$baryons (plus the corresponding antiparticles for $\bar{c}$ ). In Fig. 1-left we plot the fraction of energy taken by the hadron pair $\left(H_{Q} \bar{H}_{Q}^{\prime}\right)$ together with the relative abundance of each species in $10^{10} \mathrm{GeV} p$-air collisions. For the bottom quark (see [18]) we consider only fragmentation into $B^{-}$or $\bar{B}^{0}$ mesons, with equal frequency and a fragmentation function $D_{b}(z)=N z^{13.7}(1-z)$, where $z=E_{H} / E_{Q}$. In Fig. 1-right we plot the fraction of energy taken by the pair of $B$ mesons after the $b \bar{b}$ pair has been produced.

We have included in AIRES the production of heavy-hadron pairs in nucleon and meson collisions with incident energy above $10 \mathrm{TeV}$, and have considered only pairs carrying more 


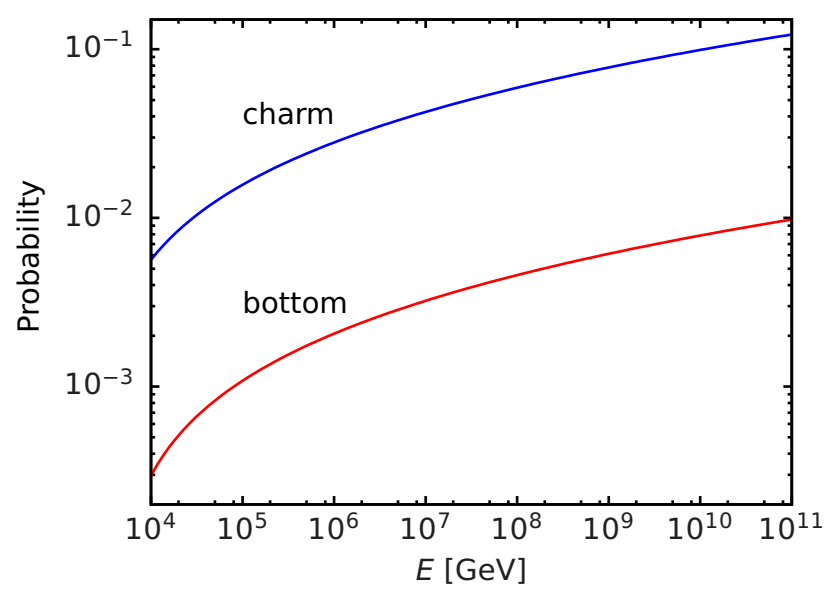

Figure 2: Probability to produce a charm or a bottom pair carrying more than $1 \%$ of the proton energy in $p$-air collisions.

than $1 \%$ of the projectile energy. These thresholds ensure the inclusion of all the effects that are relevant in air shower simulations and avoid complicating unnecesarily the procedure. A pair of energy $E_{Q \bar{Q}}=x_{F} E$ may come from a parent hadron of energy $10 E_{Q \bar{Q}}$ in a collision of $x_{F}=0.1$, or from a $1000 E_{Q \bar{Q}}$ parent for $x_{F}=0.001$. Given the steep power law observed in the cosmic ray flux, however, the contribution to the production of heavy quark pairs from small- $x_{F}$ collisions is not important.

Dividing by the total (inelastic) $p$-air cross section we obtain the probability to produce pairs of charm or bottom hadrons with $x_{F}>0.01$. In Fig. 2 we plot this probability for different incident energies between $10^{4}$ and $10^{11} \mathrm{GeV}$. The same production probability in the interactions of neutrons, charged pions and kaons with air has been assumed.

AIRES allows then the heavy hadrons either to decay or to collide with an air nucleus. In the case of a collision, we have taken the inelasticity and the interaction lengths for charm and bottom hadrons from [19] and [20], respectively. For example, a $D$ meson after a $10^{9} \mathrm{GeV}$ collision could keep around $55 \%$ of the initial energy, whereas a $B$ meson will typically exit with $80 \%$ of the incident energy after colliding with an air nucleus. In contrast, the leading meson after a $10^{9} \mathrm{GeV}$ pion collision would carry in average just $22 \%$ of the energy.

\section{Heavy-quark production and evolution in air shower development}

To study the heavy hadron production and evolution inside the shower, we have generated 10,000 vertical showers initiated by a $30 \mathrm{EeV}$ proton primary and have simulated them down 

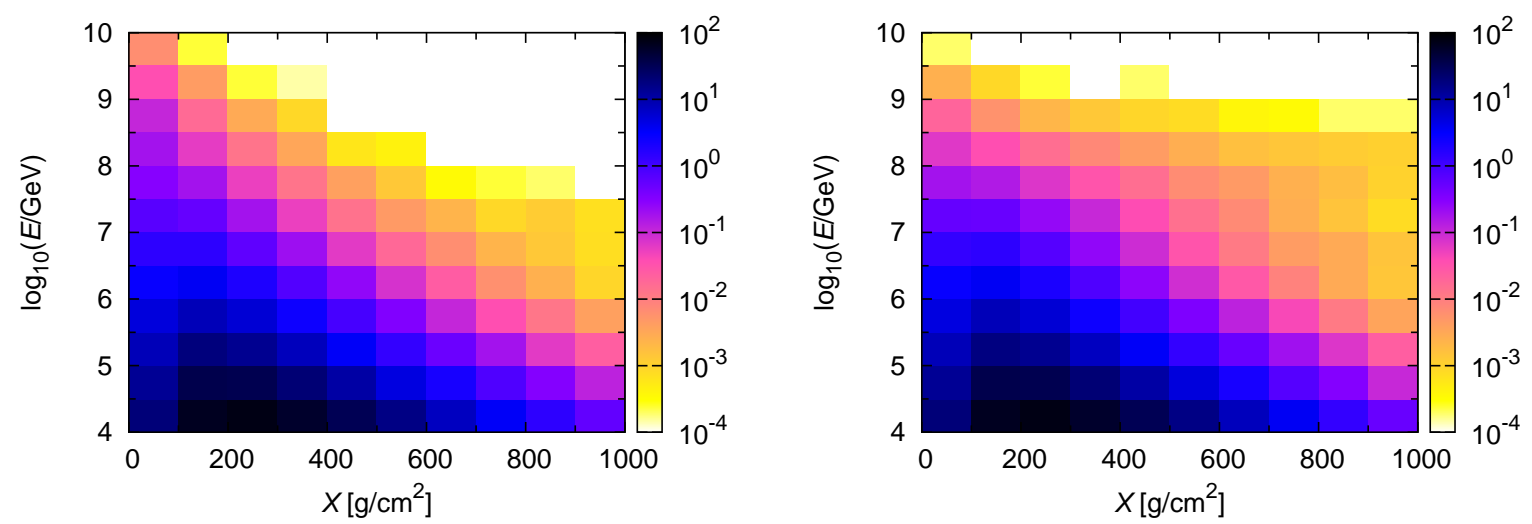

Figure 3: Average number of charm hadrons produced (left) and decayed (right) per bin of energy and atmospheric depth.

to sea level $\left(1000 \mathrm{~g} / \mathrm{cm}^{2}\right)$

In Fig. 3-left we plot the average number of charmed hadrons produced per shower in bins of $100 \mathrm{~g} / \mathrm{cm}^{2}$ and half a decade of energy. For example, from the plot it follows that around 0.19 charmed hadrons of $10^{7.5}-10^{8.0} \mathrm{GeV}$ are produced at atmospheric depths between 100 and $200 \mathrm{~g} / \mathrm{cm}^{2}$ per shower. These hadrons are produced in secondary collisions and also through the decay of $B$ mesons (most of the high-energy charmed hadrons beyond $600 \mathrm{~g} / \mathrm{cm}^{2}$ come from $B$ decays).

Adding bins we find that an average $30 \mathrm{EeV}$ shower contains 0.5 charmed hadrons of energy above $10^{8} \mathrm{GeV}$, and $6 \%$ of them are produced beyond $200 \mathrm{~g} / \mathrm{cm}^{2}$. The total energy transferred into these very energetic hadrons is $2.0 \times 10^{8} \mathrm{GeV}$, i.e., $0.7 \%$ of the energy of the primary proton. Concerning charmed-hadron decay, we plot in Fig. 3-right its depth and energy distributions. We find an average of 1.0 charmed hadrons of energy above $10^{5} \mathrm{GeV}$ decaying after $600 \mathrm{~g} / \mathrm{cm}^{2}$. They carry a total energy of $2.9 \times 10^{6} \mathrm{GeV}$ beyond that atmospheric depth.

Bottom hadrons are more rare in these showers, but their effect tends to be more radical. We plot the depth and energy distributions for their production (Fig. 4-left) and their decay (Fig. 4-right) for $30 \mathrm{EeV}$ vertical showers. We find around $38 \mathrm{~B}$ mesons of energy above $10^{8} \mathrm{GeV}$ per 1000 showers, with an average production depth of $97 \mathrm{~g} / \mathrm{cm}^{2}$. This means that only $4 \%$ of the $30 \mathrm{EeV}$ showers include such an energetic bottom hadron. Given the higher elasticity in their collisions, these $B$ mesons reach deeper in the atmosphere than charmed hadrons before they decay. In particular, we find around 16 hadrons per 1000 showers decaying at atmospheric depths beyond $600 \mathrm{~g} / \mathrm{cm}^{2}$ with energies above $10^{6} \mathrm{GeV}$, and 1 hadron of energy above $10^{7} \mathrm{GeV}$ reaching the ground at $1000 \mathrm{~g} / \mathrm{cm}^{2}$. 


\section{Tau leptons and muons}

In this section we present the frequency of tau lepton events and the average muon energy distribution from our simulation of $30 \mathrm{EeV}$ vertical proton showers.

Tau leptons are mainly produced in $D_{s}$ decays. They may introduce interesting effects because their decay length $(c \tau=87 \mu \mathrm{m})$ reaches $5 \mathrm{~km}$ at $10^{8} \mathrm{GeV}$. In Fig. 5-left (right) we plot the point and energy where they are produced (decay) inside the shower. We find 23 tau leptons of energy above $10^{7} \mathrm{GeV}$ per 1000 showers. They are produced at an average depth of $270 \mathrm{~g} / \mathrm{cm}^{2}$ and decay at $320 \mathrm{~g} / \mathrm{cm}^{2}$, in particular, $18 \%$ of them decay after $600 \mathrm{~g} / \mathrm{cm}^{2}$. For example, in the energy bin between $10^{8}$ and $10^{8.5} \mathrm{GeV}$ we obtain 2.2 tau leptons per 1000 vertical showers, with 0.8 of them reaching the ground. Therefore, the frequency of very energetic taus produced in EAS that decay near the ground is similar to the frequency of the analogous $B$-meson events.

Muons, on the other hand, are a key prediction in air-shower simulations. Although the presence of heavy hadrons will not introduce significant differences in the total number of muons at the ground level, there are other observables that may be more sensitive to these heavy hadrons. In particular, one could expect two types of effects.

- Rare events with late energy deposition from the decay of a heavy meson or a $\tau$ lepton. A $10^{8} \mathrm{GeV}$ deposition relatively near the ground would produce muons and other charged particles that could change significantly the shower profile seen in the fluorescence telescopes and/or the temporal distribution observed in the surface detectors. The fraction of these rare events is approximately $0.5 \%$.

- Leptons of PeV energies. At very high energies pions tend to collide in the air instead
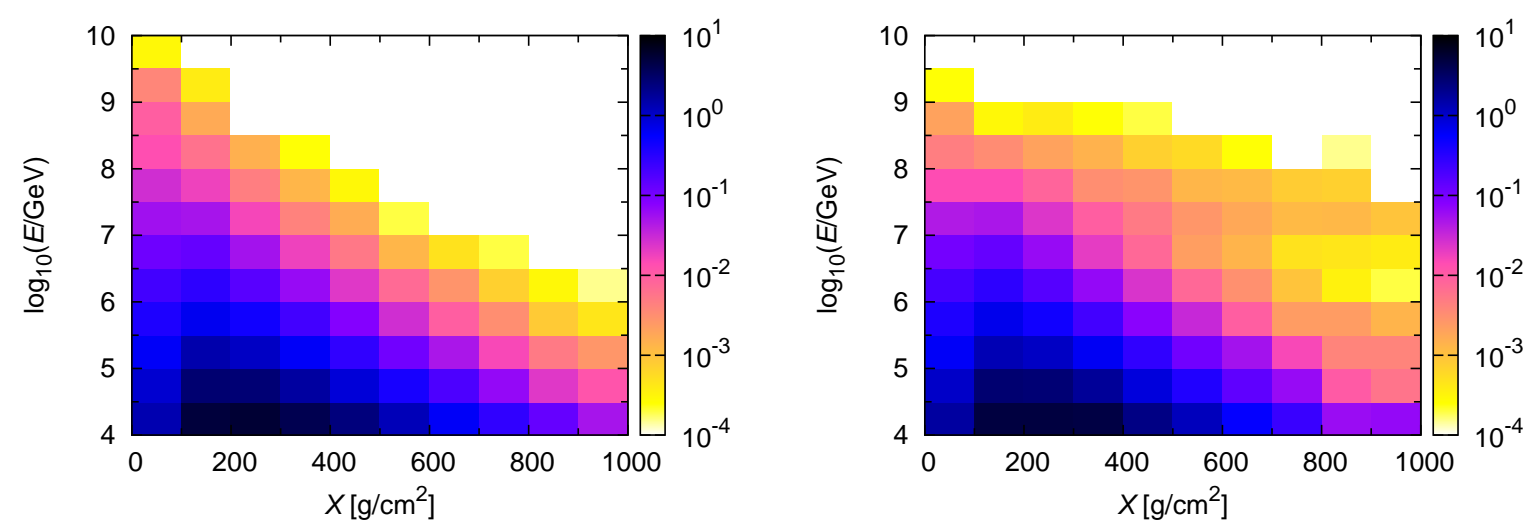

Figure 4: Average number of bottom hadrons produced (left) and decayed (right) per bin of energy and atmospheric depth. 

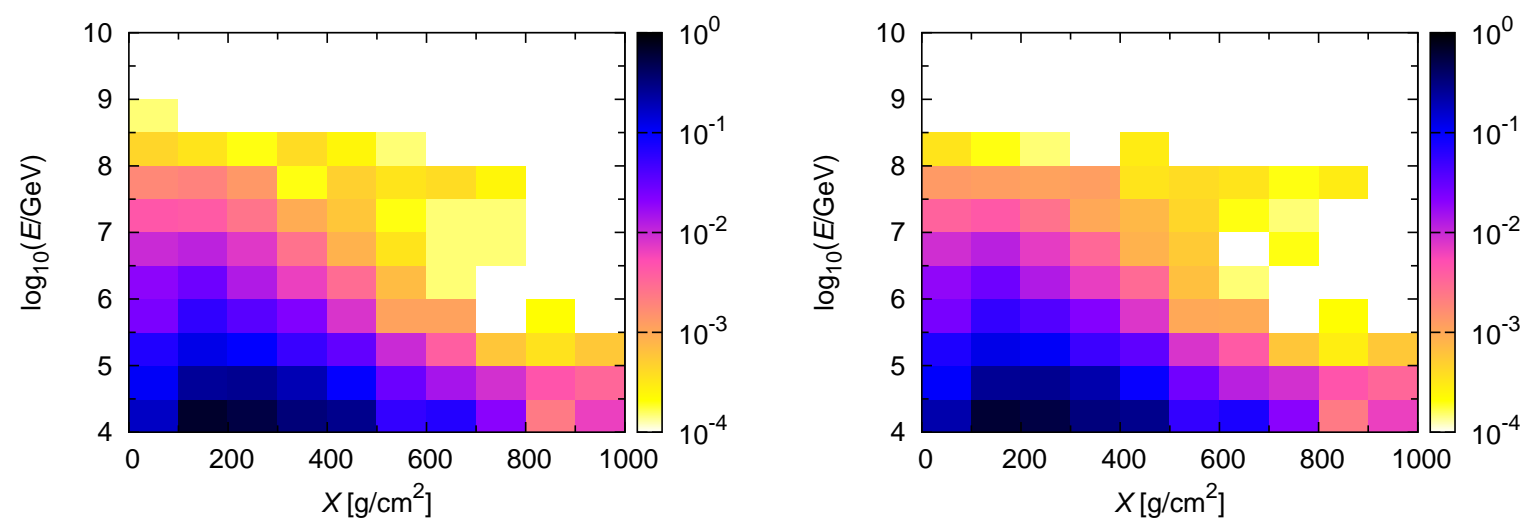

Figure 5: Average number of tau leptons produced (left) and decayed (right) per bin of energy and atmospheric depth.

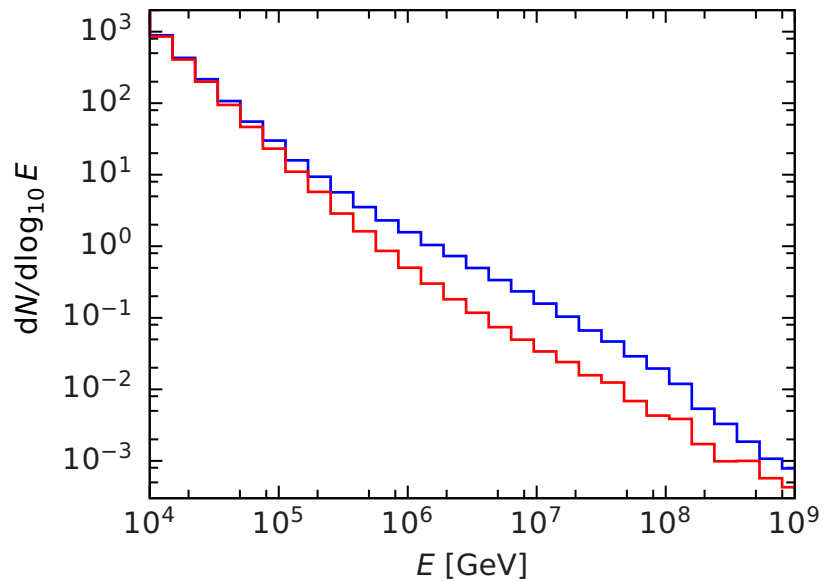

Figure 6: Average energy distribution of ground muons from a $30 \mathrm{EeV}$ proton shower simulated with (upper histogram) and without (lower histogram) the production, propagation and decay of heavy hadrons.

of decaying thus becoming a less effective source of muons [11, 12]. This shows up clearly in Fig. 6, where we plot the energy distribution of muons reaching the ground for a $30 \mathrm{EeV}$ proton primary. The distribution in showers simulated using HQIP is almost 5 times larger than the one with no heavy quark production at $\mathrm{PeV}$ energies. These very energetic muons may be observable at neutrino telescopes [21], and they could be used to estimate the correlated neutrino flux. 


\section{Summary and discussion}

At energies above $10^{8} \mathrm{GeV}$ hadrons containing a charm or a bottom quark become long lived, and it is then necessary to implement their collisions with air nuclei in air shower simulations. Although the interactions with matter of $D$ and $B$ mesons are not observable at colliders, one expects that they are much more elastic than pion or proton collisions.

The main effect of these particles would derive from their ability to transport energy deep into the atmosphere. Since the fraction of events with a very energetic heavy hadron is small, one can not expect significant differences in the features of the average shower. In particular, we have studied common observables like the lateral and the longitudinal distributions of charged particles, and in all cases we could not observe any relevant differences due to heavy quark effects. Instead, one could look for anomalous events with late energy deposition caused by their decay.

We have included both the production and the propagation of heavy hadrons in a new version of AIRES. To illustrate the performance of the code, we have simulated 10,000 vertical showers of fixed $30 \mathrm{EeV}$ energy. We find around one $D$ meson of energy above $10^{8} \mathrm{GeV}$ per 2 showers, or just one $B$ meson in this energy range per 26 showers. A few per mille of these air showers includes a $B$ meson of energy above $10^{7} \mathrm{GeV}$ hitting the ground at $1000 \mathrm{~g} / \mathrm{cm}^{2}$. The frequency of very energetic tau leptons from $D_{s}$ decays reaching large atmospheric depths is slightly higher. The uncertainty in all these rates combines a $50 \%$ variation in the production cross sections with the uncertainty in the inelasticity of heavy hadron-nucleus collisions, that is more difficult to estimate since such process can not be observed at colliders. Therefore, we think that a search for possible signals associated to these rare events should be considered even if the expected rates are small.

The results presented here point to different air shower observables that may reflect the production of heavy quarks, but a definite determination of their observability would require the generation of air showers of different energies and from different inclinations. The inclusion of heavy hadrons in AIRES opens the possibility to optimize such a search.

\section{Acknowledgments}

We would like to thank Javier Albacete, Antonio Bueno, and Alberto Gascón for useful discussions. The work has been partially supported by ANPCyT and CONICET of Argentina, by MINECO of Spain (FPA2010-16802 and Consolider-Ingenio Multidark CSD2009-00064) and by Junta de Andalucía (FQM 101, FQM 03048, FQM 6552). 


\section{References}

[1] S. J. Sciutto, AIRES, a system for air shower simulation and analysis; Proc. 27th ICRC (Hamburg), 1, 237 (2001). See also www2.fisica.unlp.edu.ar/aires.

[2] J. Knapp, D. Heck, S. J. Sciutto, M. T. Dova and M. Risse, Astropart. Phys. 19 (2003) 77.

[3] P. Abreu et al. [Pierre Auger Collaboration], Eur. Phys. J. Plus 127 (2012) 87.

[4] R. S. Fletcher, T. K. Gaisser, P. Lipari and T. Stanev, Phys. Rev. D 50, 5710 (1994); R. Engel, T. K. Gaisser, T. Stanev and P. Lipari, Proc. 26th International Cosmic Ray Conference (Utah) 1, 415 (1999).

[5] S. Ostapchenko, Nucl. Phys. Proc. Suppl., B151, 143 (2006).

[6] D. Heck, Forschungszentrum Karlsruhe Report FZKA 7366 (2008).

[7] R. Emparan, M. Masip and R. Rattazzi, Phys. Rev. D 65 (2002) 064023; J. L. Feng and A. D. Shapere, Phys. Rev. Lett. 88 (2001) 021303; J. I. Illana, M. Masip and D. Meloni, Phys. Rev. Lett. 93 (2004) 151102.

[8] G. Burdman, F. Halzen and R. Gandhi, Phys. Lett. B 417 (1998) 107; M. Kachelriess and M. Plumacher, Phys. Rev. D 62 (2000) 103006; L. A. Anchordoqui, J. L. Feng, H. Goldberg and A. D. Shapere, Phys. Rev. D 66 (2002) 103002.

[9] I. F. M. Albuquerque, G. Burdman and Z. Chacko, Phys. Rev. Lett. 92 (2004) 221802; M. Ahlers, J. Kersten and A. Ringwald, JCAP 0607 (2006) 005; M. Ahlers, J. I. Illana, M. Masip and D. Meloni, JCAP 0708 (2007) 008; L. A. Anchordoqui, A. Delgado, C. A. García Canal and S. J. Sciutto, Phys. Rev. D 77 (2008) 023009.

[10] P. Lipari, Astropart. Phys. 1 (1993) 195.

[11] G. Battistoni, C. Bloise, C. Forti, M. Greco, J. Ranft and A. Tanzini, Astropart. Phys. 4 (1996) 351; P. Gondolo, G. Ingelman and M. Thunman, Astropart. Phys. 5 (1996) 309; L. Pasquali, M. H. Reno and I. Sarcevic, Phys. Rev. D 59 (1999) 034020. C. G. S. Costa, Astropart. Phys. 16 (2001) 193.

[12] J. I. Illana, P. Lipari, M. Masip and D. Meloni, Astropart. Phys. 34 (2011) 663.

[13] P. Berghaus, T. Montaruli and J. Ranft, JCAP 0806 (2008) 003.

[14] N. N. Nikolaev and B. G. Zakharov, Phys. Lett. B 260 (1991) 414.

[15] V. P. Goncalves and M. V. T. Machado, JHEP 0704 (2007) 028. 
[16] R. Enberg, M. H. Reno and I. Sarcevic, Phys. Rev. D 78 (2008) 043005.

[17] B. A. Kniehl and G. Kramer, Phys. Rev. D 74 (2006) 037502.

[18] B. Mele and P. Nason, Phys. Lett. B 245 (1990) 635; J. Beringer et al. [Particle Data Group Collaboration], Phys. Rev. D 86 (2012) 010001.

[19] R. Barceló, J. I. Illana, M. D. Jenkins and M. Masip, Phys. Rev. D 83 (2011) 034027.

[20] A. Bueno, A. Gascón, J. I. Illana and M. Masip, JCAP 1202 (2012) 028.

[21] J. A. Aguilar et al. [ANTARES Collaboration], Astropart. Phys. 34 (2010) 179 [arXiv:1007.1777 [astro-ph.HE]]. 